${ }^{1}$ Centro de Investigación en Salud Pública y Epidemiología Clínica (CISPEC). Facultad de Ciencias de la Salud Eugenio Espejo. Universidad Tecnológica Equinoccial. Quito-Ecuador

Fuentes de financiamiento: Universidad Tecnológica Equinoccial. Conflictos de interés: Los autores declaran no tener conflictos de interés.

Recibido el 20 de marzo de 2018, aceptado el 19 julio de 2018.

Correspondencia a: Solange Vianney Núñez González Dirección: Avenida Mariscal Sucre y Mariana de Jesús, Quito, Ecuador. solangev.nunez@ute.edu.ec

\section{Mortalidad por enfermedades isquémicas del corazón en Ecuador, 2001-2016: estudio de tendencias}

\author{
SOLANGE NÚÑEZ-GONZÁLEZ ${ }^{1}$, SANDY AULESTIA-ORTIZ1, \\ EDISON BORJA-VILLACRÉS ${ }^{1}$, DANIEL SIMANCAS-RACINE ${ }^{1}$
}

\section{Mortality due to ischemic heart disease in Ecuador 2001-2016. A time trend study}

Background: Ischemic Heart Disease (IHD) is the leading cause of mortality worldwide. Aim: To analyze the evolution of mortality by IHD in Ecuador, from 2001 to 2016. Material and Methods: Analysis of Ecuador death registries elaborated by the National Statistics and Census Institute. Specific and age-adjusted mortality rates were calculated for the 2001-2016 period in Ecuador. The annual percent change $(A P C)$ and the average annual percent change (AAPC) were estimated. Changes in trends were located through joinpoint regression analysis. Results: From 2001 to 2016 there were 46,133 deaths due to IHD in Ecuador, of which $60 \%(n=27,489)$ corresponded to men. In the joinpoint regression analysis, the crude mortality rates for IHD reported an increase in the AAPC of 4.0\% (2001-2016, 95\% CI: 1.5 - 6.6). Rates adjusted for age increased with an AAPC of 3.2\% (2001-2016, 95\% CI: 0.7 - 5.8). Conclusions: Mortality due to ischemic heart disease increased in Ecuador in the period 2001-2016. Two marked periods were observed, one with a decrease followed by a significant increase, in both sexes and all age groups.

(Rev Med Chile 2018; 146: 850-856)

Key words: Cardiovascular Diseases; Ecuador; Mortality; Regression Analysis.

\section{I} as enfermedades isquémicas del corazón (EIC) ocupan la primera causa de mortalidad y años de vida potencialmente perdidos (AVPP) en países de altos y medianos ingresos; afectando a ambos sexos por igual ${ }^{1-3}$. En el año 2016, se produjeron en todo el mundo $9,48 \mathrm{mi}-$ llones de defunciones por EIC, representando más de $85,1 \%$ de todas las muertes por enfermedad cardiovascular junto con la enfermedad cerebrovascular ${ }^{2}$.

En América, países como Estados Unidos y Canadá la mortalidad por EIC ha registrado un tendencia decreciente, reportado descensos en las tasas ajustada de mortalidad en hombres, pasando de 226 defunciones por cada 100.000 habitantes en 1990 a 120 en 2010, en las mujeres de 126 defun- ciones por cada 100.000 habitantes a 76 , para los mismos años; sin embargo, estos cambios han sido menos favorables en países de América Latina ${ }^{4}$.

En Colombia, Chile, Venezuela, Argentina, Bolivia, Brasil y Paraguay, las EIC ocupan el primer lugar como causa de muerte, reportando un porcentaje de cambio de $21,6 \%, 20,7 \%, 44,5 \%$, $7,0 \%, 43 \%, 22,6 \%, 48,3 \%$, respectivamente para el período 2005-2016; por otro lado, Uruguay es el único país en reportar un porcentaje de cambio decreciente de $-5,9 \%$ para el mismo período $^{5}$. En Ecuador, las EIC han ocupado por varios años los primeros lugares dentro de las principales causas de defunción, es así que para el año 2016, las EIC fueron la primera causa de mortalidad en hombres y mujeres concentrando 
el $10,15 \%(\mathrm{n}=3.747)$ y $9,04 \%(\mathrm{n}=2.766)$ de las defunciones, respectivamente ${ }^{6}$. En los últimos años, diversas estrategias se han planteado para reducir la alta mortalidad por las EIC y lograr el objetivo de disminuir en $25 \%$ el número de muertes prematuras asociadas a las enfermedades crónicas no transmisibles, propuesto por la OMS y Organización Panamericana de la Salud (OPS) ${ }^{7}$. El presente estudio tiene por objetivo analizar e identificar la presencia de cambios en la tendencia temporal de la mortalidad por IEC en Ecuador en el período 2001 a 2016.

\section{Material y Métodos}

Se llevó a cabo un estudio ecológico de series temporales, los datos de mortalidad se obtuvieron de los registros de "Defunciones Generales" de los años 2001 a 2016, elaborados por el Instituto Nacional de Estadística y Censos (INEC) ${ }^{7}$. Este registro incluye la "causa básica" de defunción codificada a partir de la Clasificación Internacional de Enfermedades (CIE); para las defunciones entre 2001 y 2016 se seleccionaron los casos por EIC con código I20-I25 según la décima clasificación (CIE-10). Los datos poblacionales se obtuvieron de las estimaciones poblacionales de los censos 2001 y 2010 realizadas por el INEC ${ }^{9}$.

Se calcularon las tasas crudas de mortalidad para cada año, las tasas específicas por sexo y grupos de edad (30-39, 40-49, 50-59, 60-69, 70-79, igual o mayor de 80 años). Además, se calcularon las tasas ajustadas por edad para la población a través del método directo, usando el estándar de población de la Organización Mundial de la Salud $(\mathrm{OMS})^{10}$. Todas las tasas son expresadas por cada 100.000 habitantes/año. Para el cálculo de las tasas de mortalidad y con intervalos de confianza de 95\% (IC95\%) se utilizó una hoja de cálculo Microsoft Excel 2010 (Microsoft Office Professional Plus 2010).

El análisis de las tendencias se realizó mediante el modelo de regresión joinpoint o también llamado modelo segmentado de Poisson, el cual proporciona el porcentaje de cambio anual (PCA), el promedio del porcentaje de cambio anual (PPCA), y un diagrama de dispersión representada mediante trazos lineales enlazados por puntos de unión (joinpoint), permitiendo identificar el momento en el que se produce un cambio significativo de la tendencia y estimar la magnitud del aumento o el descenso en cada intervalo ${ }^{11}$.

En los modelos se establecieron un máximo de dos puntos de inflexión en cada regresión, permitiendo la selección del modelo más parsimonioso que se ajuste a los datos mediante la técnica de mínimos cuadrados ponderados, estimando su significación estadística por medio de permutaciones de Monte Carlo. La significancia estadística se fijó en un valor $\mathrm{p}<0,05$. El análisis de las tendencias se realizó a través del software gratuito 'Joinpoint' versión 4.4.011.

\section{Resultados}

Durante el período 2001-2016 se registraron 46.133 defunciones por EIC en el Ecuador, de las cuales, 59,6\% ( $n=27.489)$ corresponden a hombres, con una relación hombre-mujeres de 1,5:1. La media de edad fue de 70,9 años $( \pm 15,6)$ para los hombres y de 76,8 años $( \pm 15,0)$ para las mujeres. Las características generales de la población se muestran en la Tabla 1.

En la distribución de frecuencias de acuerdo a la clasificación CIE10, Infarto Agudo de Miocardio (I21) concentra el 91,1\% $(\mathrm{n}=42.021)$ de las defunciones, seguido por Enfermedad Cardíaca

Tabla 1. Características generales de las defunciones por enfermedades isquémicas del corazón en el Ecuador 2001-2016

\begin{tabular}{|lrr|}
\hline Característica & \multicolumn{2}{c|}{$\begin{array}{c}\text { Número y proporción de } \\
\text { defunciones }\end{array}$} \\
& $\mathbf{n}=\mathbf{4 6 . 1 3 3}$ & $\%$ \\
Sexo & & \\
$\quad$ Masculino & 27.489 & 59,59 \\
Femenino & 18.644 & 40,41 \\
Grupos etarios (años) & & \\
30-39 & 1.396 & 3,03 \\
$40-49$ & 2.74 & 5,94 \\
$50-59$ & 4.97 & 10,77 \\
60-69 & 7.704 & 16,70 \\
$70-79$ & 10.603 & 22,98 \\
$80+$ & 18.72 & 40,58 \\
Área de residencia & & \\
Urbana & 37.603 & 81,51 \\
Rural & 8.035 & 17,42 \\
Zona no delimitada & 495 & 1,07 \\
Regiones & & \\
Costa o Litoral & 25.566 & 55,42 \\
Sierra o Interandina & 19.898 & 43,13 \\
Oriental o Amazónica & 635 & 1,38 \\
Insular o Galápagos & 304 & 0,07 \\
\hline
\end{tabular}


Isquémica Crónica (I25) con 7,3\% $(\mathrm{n}=3.363)$. La distribución anual de las defunciones por CIE 10 se ilustra en la Figura 1.

La mortalidad por EIC, en números absolutos, incrementó de 2.288 defunciones en el año 2001 a 6.093 en 2016 para toda la población, las tasas crudas de mortalidad aumentaron de 74,05 a 84,41 defunciones por cada 100.000 habitantes en el período 2001-2016, en el análisis de regresión se observó un período inicial de descenso estadísticamente significativo del PCA de -3,31\% (2001-2012; $\mathrm{p}=0,007)$, seguido por un período de ascenso significativo de $28,73 \%$ anual (2012-2016; $\mathrm{p}<0,001)$. El PPCA para el período 2001-2016 fue 4,0\% (IC95\% 1,5-6,6). Las tasas ajustadas por edad también reportaron una tendencia creciente de 54,37 en 2001 a 84,90 defunciones por cada 100.000 habitantes en 2016, se registró un período inicial de descenso estadísticamente significativo del PCA del -4,74\% (2001-2012; p < 0,001), seguido por un período de ascenso significativo 28,73\% (2012-2016; $\mathrm{p}<0,001)$. El PPCA para el período 2001-2016 fue significativo 3,2\% (IC95\% $0,7-5,8)$ (Figura 2).

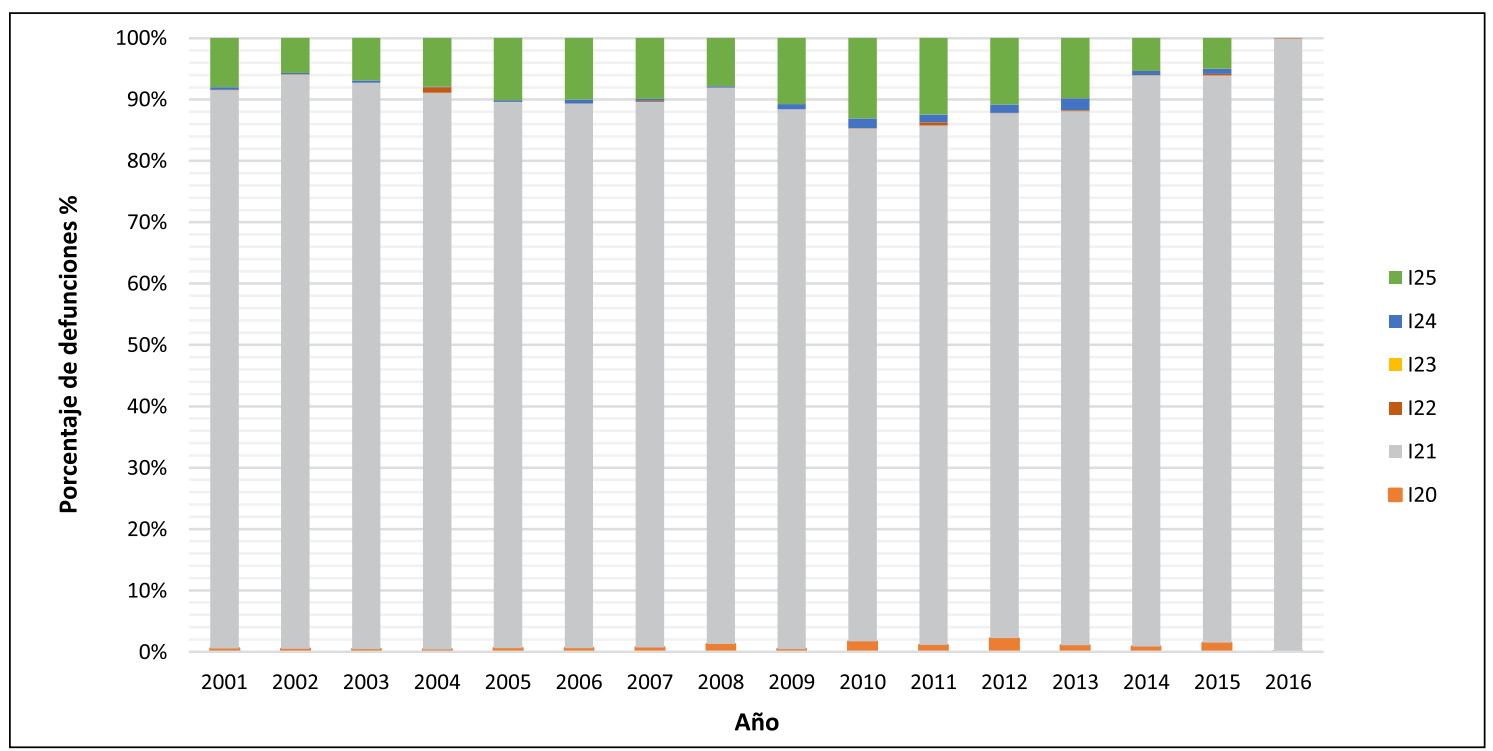

Figura 1. Porcentaje de defunciones por enfermedades isquémica del corazón según clasificación CIE-10 por año en el período 2001-2016.

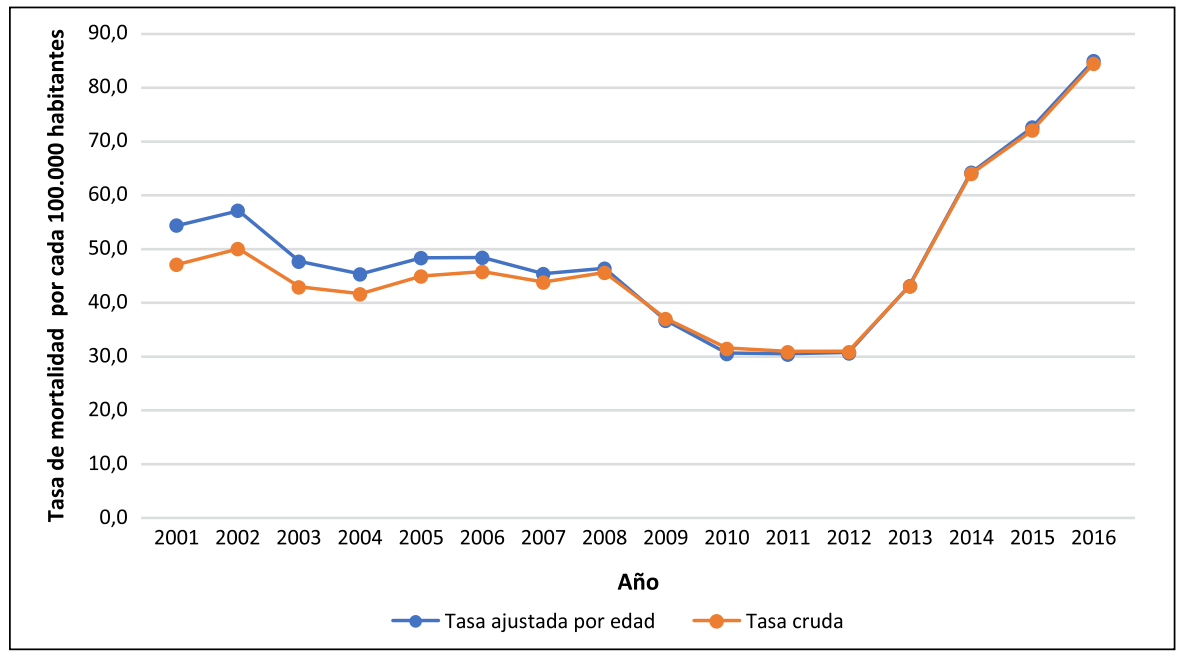

Figura 2. Tasas de mortalidad por enfermedades isquémica del corazón en toda la población, Ecuador 20012016. 

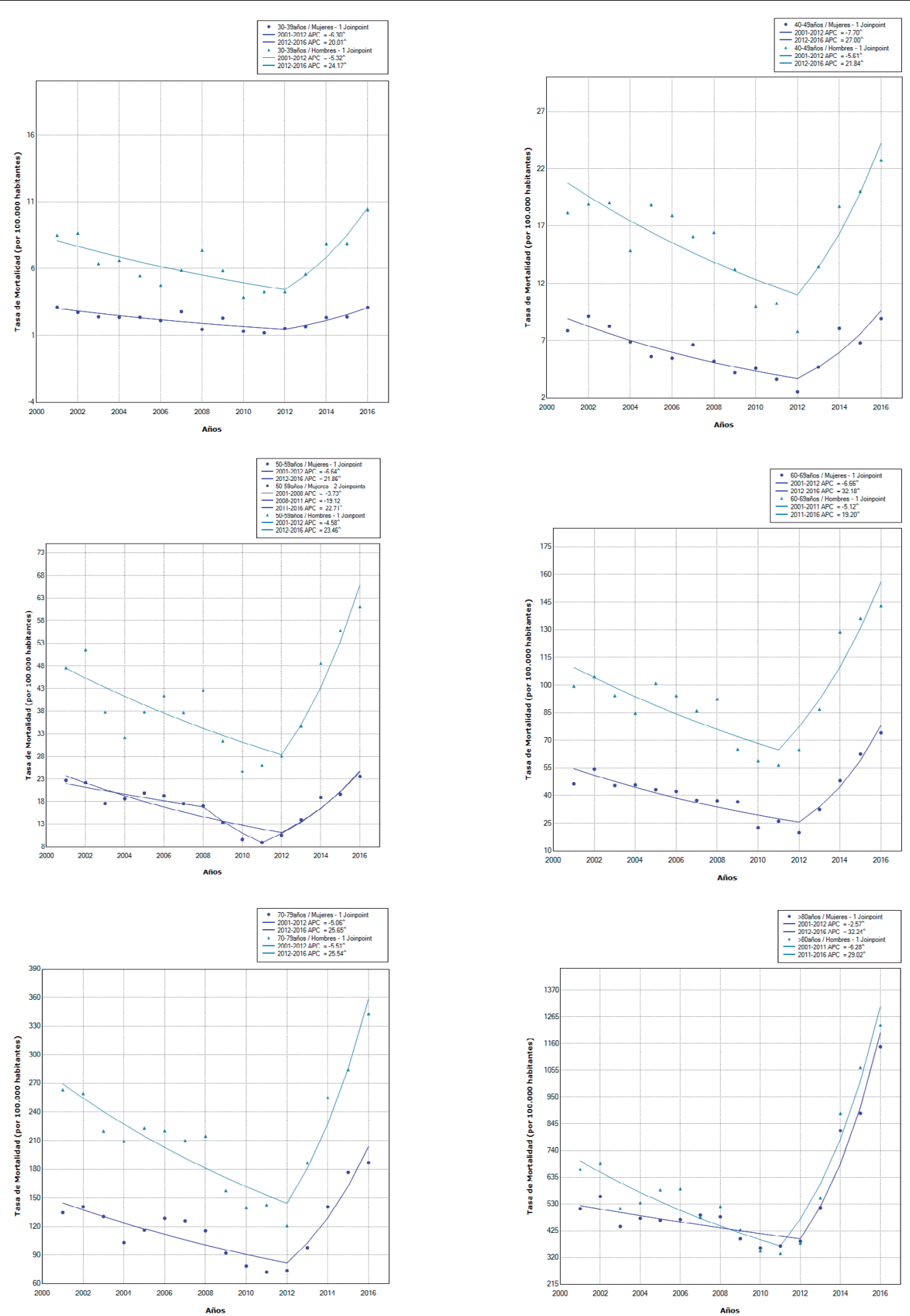

Figura 3. Análisis joinpoint de las tasas de mortalidad por enfermedades isquémica del corazón según grupos de etario y sexo en Ecuador, 2001-2016. APC = Porcentaje de Cambio Anual; ^ El PCA es significativamente distinto de $0(p<0,05)$. 
En el análisis de regresión de las tasas de mortalidad específicas por grupo etario para toda la población, todos los grupos etarios presentaron un período inicial (2001-2012) de descenso estadísticamente significativos: $30-39$ años $(-5,81 \%$; $\mathrm{p}<0,001)$; 40-49 años $(-6,33 \%$; $\mathrm{p}=0,0001) ; 50$ 59años $(-5,27 \%$; $\mathrm{p}=0,002) ; 60-69$ años $(-4,85 \%$; $\mathrm{p}=0,001) ; 70-79$ años $(-5,25 \% ; \mathrm{p}=0,0004)$; seguido por un período $(2012-2016)$ de ascenso significativo: $30-39$ años $(23,51 \%$; $\mathrm{p}=0,0007)$; 40 49 años $(22,97 \%$; p < 0,001); 50-59 años $(22,58 \%$; $\mathrm{p}=0,009) ; 60-69$ años $(26,35 \% ; \mathrm{p}=0,0001) ; 70$ 79años $(24,88 \%$; $<<0,00001)$. El grupo de $\geq 80$ años se registró un período inicial de descenso significativo del PCA del -4,97\% (2001-2011; $\mathrm{p}=0,009)$; seguido por un período de ascenso significativo del 26,91\% (2011-2016; $\mathrm{p}<0,001)$.

En el análisis según sexo, las tasas ajustadas por edad en hombres incrementaron de 70,68 defunciones por cada 100.000 habitantes en 2001 a 104,46 en 2016, el PCA presentó un descenso del $-4,85 \%$ anual en el período (2001-2012; $\mathrm{p}<0,001)$, seguido por un ascenso significativo del $29,39 \%$ anual $(2012-2016 ; \mathrm{p}<0,001)$ y registrando un PPCA de 3,1\% (2001-2016; IC95\% 0,3-5,9); en las mujeres, las tasas ajustadas presentaron el mismo patrón creciente, pasando de 39,69 defunciones por cada 100.000 habitantes en 2001 a 67,37 en 2016, en el análisis de regresión se observó un descenso del -5,53\% anual (2001-2012; p < 0,001), seguido por un ascenso significativo de $29,67 \%$ anual (2012-2016; $\mathrm{p}<0,001)$. El PPCA aumento 3,5\% (2001-2016; IC95\% 1,2-6,0). El análisis de regresión de las tasas de mortalidad según sexo por grupo etario se describe en la Figura 3.

\section{Discusión}

En el Ecuador, durante los años 2001 al 2016, las tasas mortalidad por EIC presentaron una tendencia creciente, en el análisis de regresión se observó dos períodos significativos, un inicial decreciente (2001-2012), seguido por un marcado período creciente (2012-2016), en toda la población. En el análisis según sexo y grupos de edad a partir de los 30 hasta los mayores de 80 años, se observó la misma tendencia inicial decreciente significativa seguida por un período creciente también significativo. En cuanto a la región geográfica y área de residencia, el mayor número de defunciones se registraron en la región litoral y en las áreas urbanas.

Al igual que los resultados obtenidos en el Ecuador, la mortalidad por EIC reporta una tendencia creciente en algunos países de medianos a bajos ingresos ${ }^{12-14}$; sin embargo, existe un marcado contraste con los países de altos ingresos en donde se ha reportado una tendencia decreciente, en regiones como Australasia, Europa occidental y América del Norte las tasas de mortalidad por EIC ajustadas por edad disminuyeron $51 \%, 46 \%$ y $43 \%$ respectivamente 5 . En el estudio realizado por Souza et al, en América las medianas de las tasas de mortalidad por enfermedades cardiovasculares fueron 20,6\% más altas en los países de medianos a altos ingresos y $56,7 \%$ más alto en los países medianos a bajos ingresos en comparación con los países de altos ingresos ${ }^{13}$. En nuestros hallazgos, la mortalidad por EIC fue igual en hombres y mujeres, similar a otros estudios reportados ${ }^{15-17}$.

Con respecto al análisis por grupos etarios, menos de $20 \%$ de las defunciones ocurrieron en personas $<60$ años de edad, similares resultados fueron reportados en estudios realizados a nivel mundial ${ }^{5,18}$, por encima de los 60 años se observaron los cambios más drásticos en el porcentaje de cambio anual, siendo el grupo de $\geq 80$ años el que duplico su tasa de mortalidad, similares resultados fueron repostados en un estudio realizado en la región ${ }^{5}$.

En el estudio publicado por Moran et. al, hasta el año 2010 Ecuador, Perú, y Bolivia reportaron las tasas de mortalidad ajustadas por edad por EIC más bajas junto con África subsahariana y Asia/Pacífico, con una tendencia decreciente ${ }^{5}$, en nuestro análisis se observó un marcada período decreciente para los años 2000-2012. Este período coincide con el crecimiento del ingreso presupuestario a nivel nacional, en el informe emitido por el Ministerio de Salud Pública, reportan que el monto del Presupuesto General del Estado asignado al sector salud aumentó más de $1.000 \%$, este valor, respecto a la producción del país en donde el producto interno bruto (PIB) tuvo un crecimiento promedio de $22,5 \%{ }^{19}$. Para el año 2000 el presupuesto asignado para el área de salud fue de USD 9,39 para cada ecuatoriano, mientras que para el año 2011 este presupuesto se multiplicó más de diez veces, asignándose USD 96,66 para cada ecuatoriano, triplicando el número de consultas preventivas e incrementado el número 
14,5 médicos por cada 10.000 habitantes en 2000 a 21,4 médicos en $2011^{19}$.

Sin embargo, a partir del año 2012 se observa una marcada tendencia creciente en las tasas de moralidad hasta el año 2016. En este período, la tasa de crecimiento del PIB en Ecuador registró un trascendental descenso, pasando de 5,64\% a $-1,47 \%$ respectivamente. Esta reducción fue el resultado de los bajos precios del petróleo, la apreciación del dólar y la crisis generada por al terremoto que afectó la zona costera noroccidental del país ${ }^{20}$. En el estudio realizado por Sosa et al, concluye que existe una estrecha relación temporal entre el aumento relativo de la mortalidad cardiovascular y el descenso del PIB, agregando que las crisis económicas tendrían un impacto asimilable a las catástrofes naturales, generando consecuencias sobre la salud cardiovascular ${ }^{21}$.

A partir del año 2012, se han implementado estrategias enfocadas en contrarrestar el efecto de los principales factores de riesgo cardiovascular, como la campaña "Ecuador libre de Humo de Tabaco"22, y se creó el programa "Ecuador, Ejercítate y vive sano" ${ }^{23}$, sin embargo, ninguna de estas estrategias han sido evaluadas para estimar su impacto en la población.

La principal fortaleza de este estudio fue el uso del modelo de regresión jointpoint para el análisis de las tendencias de mortalidad por EIC para el período 2001-2016, este modelo nos permite detectar cambios en la tendencia temporal estadísticamente significativos, lo cual evita la pre-especificación de períodos por parte del investigador. Cabe señalar que a pesar de las técnicas y ajustes realizados en este trabajo los resultados no permiten establecer una relación causal entre las tendencias observadas y los posibles factores clínicos asociados.

Con respecto a las bases de datos empleadas, el Instituto de Métricas y Evaluación de la Salud de la Universidad de Washington (IHME por sus siglas en inglés) calificó la calidad de los datos del registro del INEC para el período 1980-2016 con cuatro de cinco estrellas, como se informa en el estudio Global Burden of Disease 2016 ${ }^{2}$, lo que permite tener confianza en la calidad de los datos utilizados en este estudio. Sin embargo, con respecto al tipo de registro, de acuerdo a la codificación CIE10, consideramos que podrían existir problemas potenciales en la fiabilidad, lo cual podría introducir un sesgo de atribución, como se ha reportado en otros estudios ${ }^{24}$.
La falta de información sobre la incidencia de EIC en Ecuador nos impide identificar posible avance en la salud pública, sin embargo, el análisis temporal de la mortalidad por grupos etarios y sexo representa una primera estimación del riesgo general de la población ${ }^{25}$.

\section{Conclusión}

La mortalidad por las enfermedades isquémicas del corazón ha incrementado en Ecuador en el período 2001-2016. Se observaron dos períodos marcados, uno decreciente seguido por un creciente significativo, en ambos sexos y todos los grupos etarios incluidos en este estudio. Estos resultados alertan a la población ecuatoriana, quienes deben modificar aquellos hábitos que han probado disminuir el riesgo de mortalidad; estimulan a los investigadores a profundizar en estos hallazgos para encontrar intervenciones eficientes y efectivas, así como factores específicos poblacionales que contribuyan a la toma de decisiones en salud; e invitan a las autoridades sanitarias a enfatizar esfuerzos, replantear estrategias y/o crear nuevas intervenciones en salud que logren disminuir, o al menos estabilizar, esta tendencia.

\section{Referencias}

1. World Health Organization. World Heart Day 2017 [Internet]. [citado el 12 de enero de 2018]. Disponible en: http://www.who.int/cardiovascular_diseases/worldheart-day-2017/en/

2. Abajobir AA, Abbafati C, Abbas KM, Abd-Allah F, Abera SF, Aboyans V, et al. Global, regional, and national age-sex specific mortality for 264 causes of death, 19802016: a systematic analysis for the Global Burden of Disease Study 2016. Lancet 2017; 390 (10100): 1151-210.

3. Institute for Health Metrics and Evaluation. Country Profiles. [Internet]. [citado el 6 de junio de 2018]. Diponible en: http://www.healthdata.org/results/country-profiles.

4. Cooper R, Cutler J, Desvigne-Nickens P, Fortmann SP, Friedman L, Havlik R, et al. Trends and Disparities in Coronary Heart Disease, Stroke, Findings of the National Conference on Cardiovascular Disease Prevention. Circulation 2000; 102: 3137-47.

5. Moran AE, Forouzanfar MH, Roth GA, Mensah GA, Ezzati M, Murray CJL, et al. Temporal trends in ischemic heart disease mortality in 21 world regions, 1980 to 
2010: the Global Burden of Disease 2010 study. Circulation 2014; 129 (14): 1483-92.

6. Instituto Nacional de Estadística y Censos. Estadísticas Vitales Registro Estadístico de Nacidos vivos y Defunciones 2016 [Internet]. [citado el 2 de enero de 2018]. Disponible en: http://www.ecuadorencifras.gob. ec/documentos/web-inec/Poblacion_y_Demografia/ Nacimientos_Defunciones/2016/Presentacion_Nacimientos_y_Defunciones_2016.pdf

7. WHO. Plan de acción mundial para la prevención y el control de las enfermedades no transmisibles 2013-2020. Who. 2013. 44 p.

8. Instituto Nacional de Estadística y Censos. Nacimientos y Defunciones. Bases de Datos - Defunciones Generales [Internet]. [citado el 2 de enero de 2018]. Disponible en: http://www.ecuadorencifras.gob.ec/nacimientos-defunciones/

9. Instituto Nacional de Estadística y Censos. Población y Demografía [Internet]. [citado el 2 de enero de 2018]. Disponible en: http://www.ecuadorencifras.gob.ec/ censo-de-poblacion-y-vivienda/

10. Ahmad OB, Boschi-Pinto C, López AD. Age standardization of rates: a new WHO standard. GPE Discuss Pap Ser 2001;(31):1-14. [Internet]. [citado el 7 de enero de 2018]. Disponible en: http://www.who.int/healthinfo/ paper31.pdf.

11. Kim HJ, Fay MP, Feuer EJ MD. Permutation tests for jointpoint regression with applications to cancer rates. Stat Med 2000; 19 (April 1998): 335-51.

12. Sánchez-Arias A, Bobadilla-Serrano M, Dimas-Altamirano B, Gómez-Ortega M, González-González G. Enfermedad cardiovascular: primera causa de morbilidad en un hospital de tercer nivel. Rev Mex Cardio 2016; 27 s3: 98-102.

13. De Souza MDFM, Gawryszewski VP, Orduñez P, Sanhueza A, Espinal MA. Cardiovascular disease mortality in the Americas: Current trends and disparities. Heart 2012; 98 (16): 1207-12.

14. Roselló M, Guzmán S. Evolución de la mortalidad por enfermedad isquémica del corazón e infarto agudo del miocardio en Costa Rica, 1970-2001. Rev Panam Salud Pública 2004; 16 (5): 295-301.

15. Gouvinhas C, Severo M, Azevedo A, Lunet N. World- wide patterns of ischemic heart disease mortality from 1980 to 2010. Int J Cardiol 2014; 170 (3): 309-14.

16. Mikkola TS, Gissler M, Merikukka M, Tuomikoski P, Ylikorkala O. Sex Differences in Age-Related Cardiovascular Mortality. Onland-Moret NC, editor. PLoS One 2013; 8 (5): e63347.

17. Monroy ÓV, Aldatz FSB, Guerra AFR, Verdejo J, Bello MÁM, Violante R, et al. Morbilidad y mortalidad de la enfermedad isquémica del corazón y cerebrovascular en México. 2005. Arch Cardiol Mex 2007; 77 (1): 31-9.

18. Roth GA, Johnson C, Abajobir A, Abd-Allah F, Abera SF, Abyu G, et al. Global, Regional, and National Burden of Cardiovascular Diseases for 10 Causes, 1990 to 2015. J Am Coll Cardiol 2017; 70 (1): 1-25.

19. Ministerio de Salud Pùblica. Datos esenciales de salud 2000-2010 [Internet]. [citado el 8 de febrero de 2018]. Disponible en: http://www.salud.gob.ec/wp-content/ uploads/downloads/2013/05/Datos-esenciales-de-salud-2000-2010.pdf.

20. Banco Mundial. Ecuador: panorama general [Internet]. [citado el 1 de marzo de 2018]. Disponible en: http:// www.bancomundial.org/es/country/ecuador/overview\#1.

21. Sosa MI, Mtsac L, Racki M, Khoury M, Villarreal R, Cestari G. Crisis económico-financieras en la Argentina: ¿un nuevo factor de riesgo de mortalidad cardiovascular? Revista Argentina de Cardiología 2012; 137-44.

22. Ministerio de Salud. Ministerio de Salud promueve ambientes no contaminados con humo de tabaco. [Internet]. [citado el 1 de marzo de 2018]. Disponible en: http://www.salud.gob.ec

23. Ministerio del Deporte. Muévete Ecuador iniciará en Cuenca. [Internet]. [citado el 10 de marzo de 2018]. Diponible en: http://www.deporte.gob.ec/muevete-ecuador-iniciara-en-cuenca/

24. Chen L, Walker S, Tong S. The impact of the variation in death certification and coding practices on trends in mortality from ischaemic heart disease. Aust Health Rev 2002; 25 (4): 189-97.

25. Cayuela A, Cayuela L, Rodríguez-Domínguez S, González A, Moniche F. Analysis of cerebrovascular disease mortality trends in Andalusia (1980-2014). Neurologia 2017. pii: S0213-4853(17)30025-7. 